\title{
Medial Patellofemoral Ligament Reconstruction for Patellar Dislocation due to Rupture of the Medial Structures after Total Knee Arthroplasty : A Case Report and Review of the Literature
}

\author{
Tomohiro Goto, Daisuke Hamada, Toshiyuki Iwame, Naoto Suzue, \\ Makoto Takeuchi, Hiroshi Egawa, and Koichi Sairyo \\ Department of Orthopedics, Institute of Health Biosciences, the University of Tokushima Graduate \\ School, 3-18-15 Kuramoto, Tokushima, Japan
}

\begin{abstract}
Patellar dislocation is a well-recognized major complication after total knee arthroplasty (TKA). Treatment of this injury is determined according to the cause of the dislocation. In particular, proximal realignment, distal realignment, and lateral retinaculum release are options if patellar instability is not caused by prosthetic malposition. Here we report a case of patellar dislocation following TKA due to rupture of the medial structures that was treated by medial patellofemoral ligament reconstruction and lateral retinacular release. In addition, we provide a brief review of the related literature. J. Med. Invest. 61 : 409-412, August, 2014
\end{abstract}

Keywords : TKA (total knee arthroplasty), patellar dislocation, MPFL (medial patellofemoral ligament), reconstruction

\section{INTRODUCTION}

Despite developments in modern prosthetic designs and sophisticated surgical techniques, patellofemoral problems remain common complications of total knee arthroplasty (TKA) that require major revision surgery. Several factors have been implicated as causes of patellar subluxation or dislocation after TKA, such as malposition of the prosthesis, a poorly restored postoperative mechanical axis, component design, improper patellar preparation, and soft tissue imbalance (1). When patellar instability is attributed to prosthetic malposition, revision surgery should be considered (2). Otherwise, proximal

Received for publication February 26, 2014 ; accepted March 26, 2014.

Address correspondence and reprint requests to Daisuke Hamada, Department of Orthopedics, Institute of Health Biosciences, the University of Tokushima Graduate School, 3-18-15 Kuramoto, Tokushima 770-8503, Japan and Fax : +81-88-6331780. realignment, distal realignment, and lateral retinaculum release are suitable options (1). In the primary patellar dislocation, reconstruction of the medial patellofemoral ligament (MPFL) is an effective procedure for stabilizing a dislocated or subluxated patella (3). However, only a few studies have reported successful MPFL reconstruction for patellar subluxation or dislocation after TKA without prosthetic malposition $(4,5)$.

Here we report a case of patellar dislocation following TKA due to rupture of the medial structures that was treated by MPFL reconstruction and lateral retinacular release. In addition, we provide a brief review of the related literature.

\section{CASE PRESENTATION}

A 78-year-old woman with mild dementia was referred to us for surgery of advanced osteoarthritis 
of the left knee. Physical examination revealed a decreased range of motion from $20^{\circ}$ to $100^{\circ}$, and preoperative radiographs showed advanced degenerative arthritis of the left knee (Figure 1a-c). The preoperative femorotibial angle was $181^{\circ}$. The patient had undergone posterior stabilized TKA (Scorpio NRG ; Stryker, Mahwah, NJ). Exposure was obtained through a medial parapatellar approach and the patella was resurfaced with $8 \mathrm{~mm}$-thick polyethylene. Optimal patellar tracking was confirmed by the surgeon after implantation using the "no thumb test', which confirm the patellar tracking without holding the patella using surgeon's thumb. Lateral release was not performed in this surgery. The intraoperative range of motion improved from $20-100^{\circ}$ to $5-125^{\circ}$.

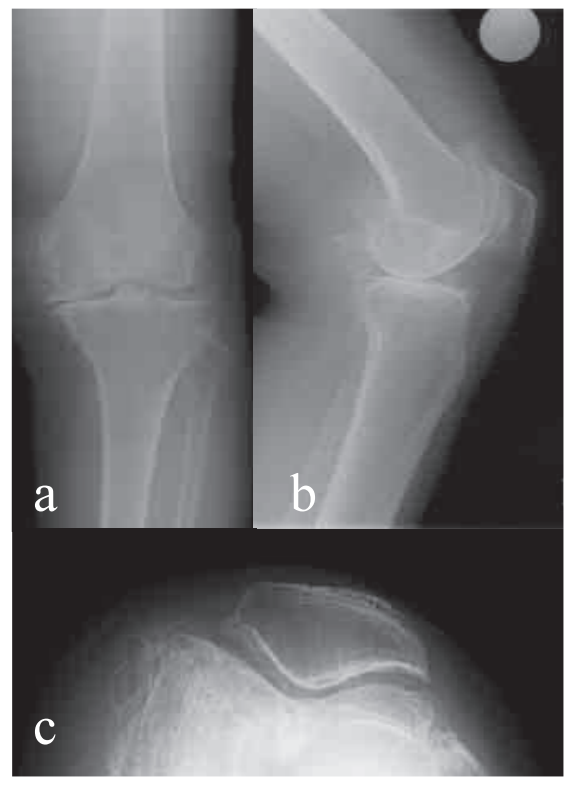

Figure 1 : Preoperative radiographs showing advanced degenerative arthritis of the left knee. (a) Anteroposterior view, (b) lateral view, and (c) skyline view.
The patient experienced several giving-way episodes after surgery and presented with partial wound disruption 4 days after surgery. Even after resuture, a faint bloody effusion from the wound was observed. The extension lag in the left knee gradually worsened after each giving-way episode. Four weeks after surgery, she presented with patellar dislocation of the left knee (Figure 2d), which was easily reproduced at $40^{\circ}$ of flexion, and vastus medialis was highly atrophic.

We assessed the cause of the dislocation to determine the treatment. Postoperative anteroposterior radiography revealed a well-restored mechanical axis with a femorotibial angle of $174^{\circ}$ (Figure 2a), suggesting that malalignment of the lower limb was not the cause of dislocation. Although lateral view radiography demonstrated a slightly oversized femoral component (Figure 2b), we did not consider it to be a critical factor for dislocation, and skyline radiographs showed an optimally placed patellar component (Figure 2c). Computed tomography revealed that the femoral component was placed at $2.9^{\circ}$ of internal rotation to the transepicondylar axis and that the anteroposterior axis of the tibial component was parallel to that of the tibia (Figure $2 \mathrm{e}-\mathrm{g}$ ). These results indicated correct placement of each prosthesis ; thus, neither malpositioning nor malrotation was the cause of the dislocation.

To stabilize the patella, we performed MPFL reconstruction with lateral retinaculum release, because conservative treatment was less reliable option in such cases. Exposure was obtained through the same incision used for TKA. A chronically ruptured medial parapatellar arthrotomy extending from the proximal patella to the tibial tuberosity seemed to have developed as the result of patellar dislocation. A 22-cm autologous semitendinosus

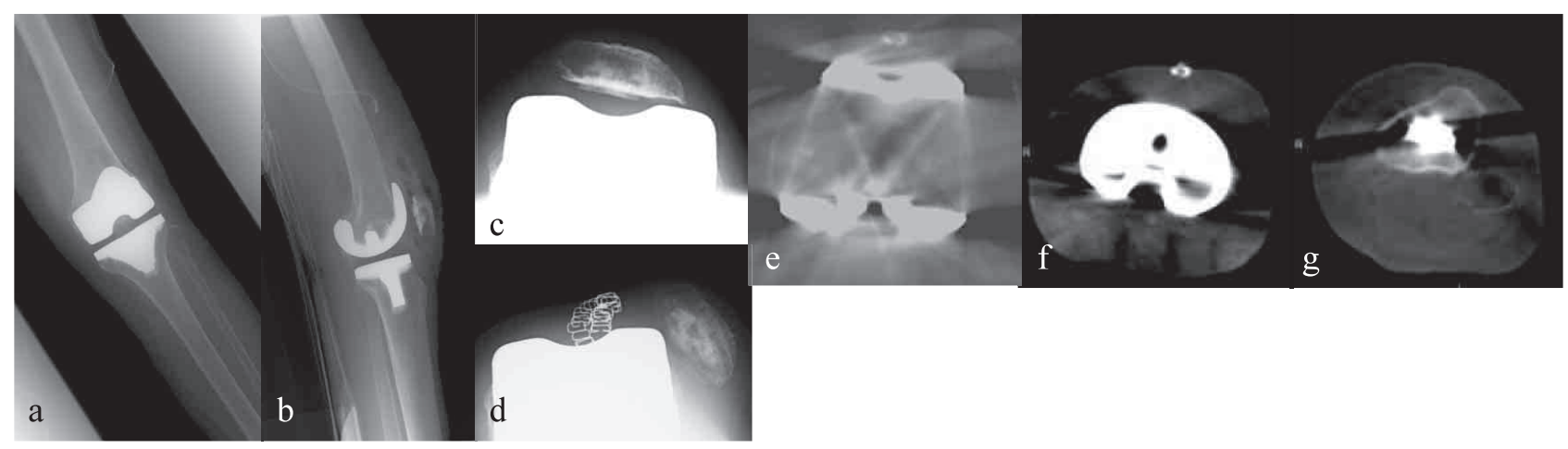

Figure 2 : Postoperative radiographs and computed tomography images showing patellar dislocation of the left knee. (a) Anteroposterior view, (b) lateral view, (c) skyline view, (d) skyline view at dislocation, (e) rotational position of the femoral component, (f) tibial component, and $(\mathrm{g})$ anteroposterior axis of the tibia. 
tendon graft was harvested with an open tendon stripper. The loop side of the double-stranded tendon graft was fixed through the femoral tunnel using an Endobutton CL (Smith \& Nephew Endoscopy, Andover, MA). The insertion point of the guide wire was placed between the medial epicondyle and adductor tubercle, and the guide wire was inserted into the lateral cortex. The guide wire was then overdrilled using a 6 - $\mathrm{mm}$ diameter cannulated drill. The lateral femoral cortex was also drilled through using the Endobutton drill. The graft was passed through the tunnel, and the Endobutton loop was flipped outside the lateral cortex. The open side of the graft was passed subcutaneously and sutured onto the proximal one-third and center of the patellar surface at $30^{\circ}$ of knee flexion (Figure $3 \mathrm{a}$ ). We also added a $4-\mathrm{cm}$ lateral release and repaired the ruptured medial parapatellar arthrotomy. Optimal patellar tracking was confirmed throughout the range of motion.

The knee was immobilized for the first 2 weeks after surgery, and range of motion exercise was started from 3 weeks after surgery. Gait exercise was permitted with an elastic brace, and weight bearing was allowed as tolerated from 3 weeks after surgery. No apprehension sign or dislocation of the patella was evident after surgery. Postoperative anteroposterior and lateral radiography showed correct fixation of the Endobutton (Figure 3b, c) and skyline radiographs showed proper positioning of the patella on the groove of the femoral component (Figure 3d).

At the 1-year follow up, the patient was able to walk with the aid of a walker without any complaints of knee pain or subluxation. The active range of motion was $10-110^{\circ}$ and no patellofemoral complications were evident.

\section{DISCUSSION}

Assessing the causes of patellar instability is important for determining appropriate treatment (2). In our case, rotational alignment of the femoral and tibial components was within the normal range. Although the patellar appeared to be slightly underresected, its composite thickness was $23 \mathrm{~mm}$, which was thinner than its preoperative thickness of 25.3 $\mathrm{mm}$. In addition, no problems were noted regarding intraoperative patellar tracking in primary TKA, suggesting there were no prosthetic issues. Therefore, we decided to treat the dislocation by realigning the patella.

Of the available patella-stabilizing options, we selected MPFL reconstruction. Although distal realignment of the patella has been reported to be effective for patellar subluxation after TKA (6), it is also a more invasive treatment, especially in elderly patients. Furthermore, an increased frequency of complications such as tibial trabecular fracture and skin necrosis has been reported for this type of osteotomy in TKA (7).

The MPFL has attracted attention as the primary medial restraint for tethering the patella $(8,9)$, and MPFL reconstruction has been proposed as the primary surgical choice for chronic patellar instability in the primary patellar dislocation (10). MPFL reconstruction for patellar maltracking after primary TKA was first reported by Asada et al. (4). Later, Matsushita et al. combined this procedure for a case of TKA showing persistent patellar dislocation during surgery (11). In each case, the dislocated patella was successfully stabilized by this method. Recently, van Gennip et al. concluded that patellar maltracking after primary or revision TKA without malrotation can be effectively treated by MPFL reconstruction

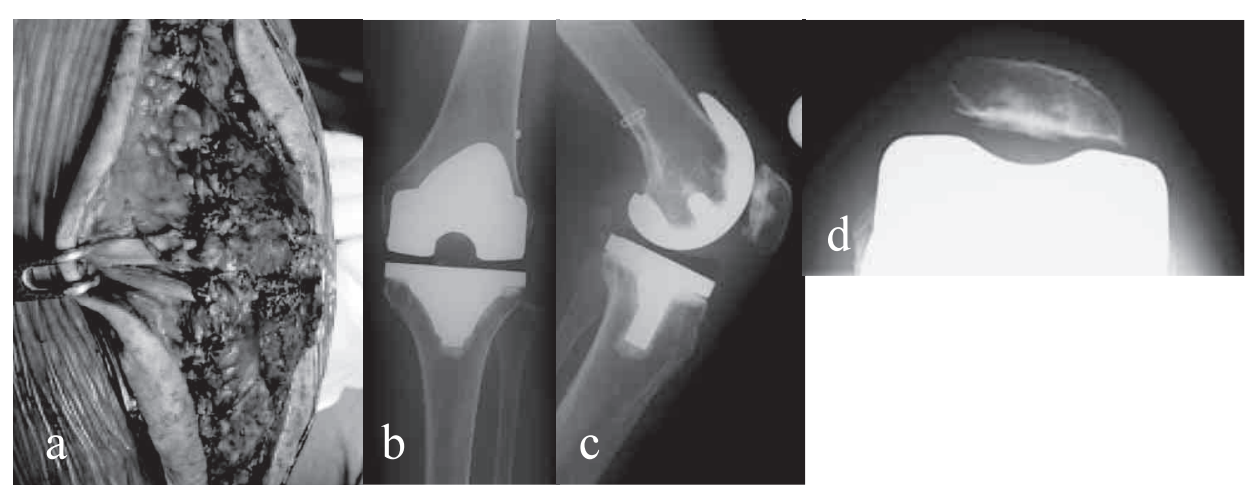

Figure 3 : Medial patellofemoral ligament (MPFL) reconstruction for patellar dislocation. (a) The open side of the graft was sutured onto the proximal one-third and center of the patellar surface. (b) Anteroposterior, (c) lateral, and (d) skyline view radiographs showing MPF. 
combined with lateral release (5). They noted the need for additional tibial tuberosity transfer in only a limited number of cases and reported that the cause of patellar instability is unclear in most cases with no component malrotation or bony malalignment. Asada et al. speculated that the patellar dislocation in their case was caused by a tear of the medial retinaculum, including the MPFL, due to knee sprain after primary TKA (4). In our case, we confirmed rupture of the medial structures that had been closed after implantation. The excessive stress that developed after multiple giving-way episodes during the early postoperative period might have caused this rupture. Therefore, close attention should be paid to preventing giving-way in patients with weakness of the vastus medialis obliquus.

Carmont et al. reported a case of MPFL reconstruction for recurrent patellar dislocation after patellofemoral joint arthroplasty (12). In their case, they initially failed to stabilize the patella by medial capsular repair and lateral retinacular release before successful MPFL reconstruction. Interestingly, Nakajima $e t$ al. also reported failure of lateral release and patellar resurfacing (6). These results suggest that MPFL reconstruction might be more promising than medial capsular repair or lateral retinacular release for stabilizing the patella.

In conclusion, the present case suggests that MPFL reconstruction and lateral retinaculum release provide a successful means of treating patellar dislocation after TKA due to rupture of the medial structures without prosthetic malposition. Furthermore, if stretching or rupture of the medial structures is attributed to dislocation or subluxation of the patella, it might be better to perform MPFL reconstruction in addition to capsular repair or lateral retinacular release.

\section{CONFLICT OF INTEREST}

All authors declare no conflicts of interest.

\section{REFERENCES}

1. Malo M, Vince KG : The unstable patella after total knee arthroplasty : etiology, prevention, and management. J Am Acad Orthop Surg 11: 364-371, 2003
2. Eisenhuth SA, Saleh KJ, Cui Q, Clark CR, Brown TE : Patellofemoral instability after total knee arthroplasty. Clin Orthop Relat Res 446 : 149-160, 2006

3. Buckens CFM, Saris DBF : Reconstruction of the medial patellofemoral ligament for treatment of patellofemoral instability : a systematic review. Am J Sports Med 38 : 181-188, 2010

4. Asada S, Akagi M, Mori S, Hamanishi C : Medial patellofemoral ligament reconstruction for recurrent patellar dislocation after total knee arthroplasty. J Orthop Sci 13 : 255-258, 2008

5. Van Gennip S, Schimmel JJP, van Hellemondt GG, Defoort KC, Wymenga AB : Medial patellofemoral ligament reconstruction for patellar maltracking following total knee arthroplasty is effective. Knee Surg Sports Traumatol Arthrosc. Epub ahead of print, 2012

6. Nakajima A, Watanabe H, Rokkaku T, Koda M, Yamada T, Murakami M : The Elmslie-Trillat procedure for recurrent patellar subluxation after total knee arthroplasty. J Arthroplasty 25 : 1170.e1-5, 2010

7. Piedade SR, Pinaroli A, Servien E, Neyret P : Tibial tubercle osteotomy in primary total knee arthroplasty : A safe procedure or not? Knee $15:$ 439-446, 2008

8. Conlan T, Garth WP, Lemons JE : Evaluation of the medial soft-tissue restraints of the extensor mechanism of the knee. J Bone Joint Surg Am 75 : 682-693, 1993

9. Desio SM, Burks RT, Bachus KN : Soft tissue restraints to lateral patellar translation in the human knee. Am J Sports Med 26 : 59-65, 1998

10. Smith TO, Walker J, Russell N : Outcomes of medial patellofemoral ligament reconstruction for patellar instability : a systematic review. Knee Surg Sports Traumatol Arthrosc 15 : 13011314, 2007

11. Matsushita T, Kuroda R, Kubo S, Mizuno K, Matsumoto T, Kurosaka M : Total knee arthroplasty combined with medial patellofemoral ligament reconstruction for osteoarthritic knee with preoperative valgus deformity and chronic patellar dislocation. J Arthroplasty 26 : 505.e17-20, 2011

12. Carmont MR, Crane T, Thompson P, Spalding $\mathrm{T}$ : Medial patellofemoral ligament reconstruction for subluxating patellofemoral arthroplasty. Knee $18: 130-132,2011$ 